Conclusion The JNK signalling transduction pathway may play important roles in the pathogenesis of NEC. ITF may protect the mucosa from injury by inhibiting JNK signalling transduction pathway.

\section{Paediatric Surgery}

\section{PO-0924b EFFECT AND SIGNIFICANCE OF ITF ON P38 SIGNALLING TRANSDUCTION PATHWAY IN NEONATAL RAT NECROTIZING ENTEROCOLITIS}

1J Zhao, ${ }^{2} X$ Fu. ${ }^{1}$ Department of Pediatric Surgery, The First Hospital of Jilin University, Changchun, China; ${ }^{2}$ Nursing Department, The Third Hospital of Jilin University, Changchun, China

10.1136/archdischild-2014-307384.1545

Purpose To explore whether one of the mechanisms of intestinal trefoil factor (ITF) in the treatment of necrotizing enterocolitis (NEC) is related to the P38 pathway.

Methods Forty wistar rats, one day after birth, were equally divided into four groups: normal control group, NEC model group, NEC+ITF group and NEC+P38Inhibitor (SB203580) group. The rats were fed for 3 days and killed on the 4th day. The ileocecum were obtained for histological examination by HE staining, P38 protein expression detection by Western-Blot and P38 gene transcripts detection by RT-PCR.

Results The pathological lesions showed that intestinal necrosis of NEC+ITF group and NEC+Inhibitor group was lighter than that of NEC model group. The level of P38 protein expression and gene transcripts in NEC model group was obviously higher than that in normal control group $(\mathrm{p}>0.05)$. Compared with NEC Model group, the level of P38 expression and gene transcripts was obviously decreased in NEC+ITF group and NEC + Inhibitor group $(\mathrm{p}>0.05)$.

Conclusion One of the pathogenesis for NEC may be related to P38 signalling transduction pathway, ITF may ameliorate intestinal inflammation and protect the mucosa from injury by inhibiting P38 signalling transduction pathway.

\section{Pharmacology}

\section{P0-0925 CHILDREN AND YOUNG PEOPLE'S EXPERIENCES OF ADVERSE DRUG REACTIONS AND PHARMACOVIGILANCE IN THE UK}

\footnotetext{
${ }^{1} \mathrm{~J}$ Arnott, ${ }^{2} \mathrm{H}$ Hesselgreave, ${ }^{3} \mathrm{AJ}$ Nunn, ${ }^{4} \mathrm{M}$ Peak, ${ }^{5} \mathrm{M}$ Pirmohamed, ${ }^{6} \mathrm{RL}$ Smyth, ${ }^{7} \mathrm{MA}$ Turner, ${ }^{8} \mathrm{~B}$ Young. ${ }^{1}$ Health Children's Nursing Research Unit, University of Central Lancashire Alder Hey Children's NHS Foundation Trust, Preston Liverpool, UK; ${ }^{2}$ Centre for Medical Education Research School of Medicine Pharmacy and Health, Durham University, Durham, UK; ${ }^{3}$ Department of Women's and Children's Health Institute of Translational Medicine, University of Liverpool Alder Hey Children's NHS Foundation Trust, Liverpool, UK; ${ }^{4}$ School of Health Research and Development, University of Central Lancashire Alder Hey Children's NHS Foundation Trust, Preston Liverpool, UK; ${ }^{5}$ Department of Molecular and Clinical Pharmacology, University of Liverpool, Liverpool, UK; ${ }^{6}$ Institute of Child Health, University College London Great Ormond Street Hospital, London, UK; ${ }^{7}$ Department of Women's and Children's Health Institute of Translational Medicine, University of Liverpool Liverpool Women's NHS Foundation Trust, Liverpool, UK; ${ }^{8}$ Institute of Psychology Health and Society, University of Liverpool, Liverpool, UK
}

10.1136/archdischild-2014-307384.1546
Background Children suffer adverse drug reactions (ADRs) yet there is no research on their experiences and no guidance for practitioners on how to communicate with children and young people in this context.

Aims To investigate children and young people's experiences of ADRs to inform communication strategies within paediatric settings.

Methods Semi-structured qualitative interviews with children and young people who had experienced a suspected ADR. Interpretive analysis informed by the constant comparative approach. Results Interviews with 20 children and young people aged 618 years suggest variable experiences. Key themes include poor awareness of potential ADRs; feeling frightened and confused by the symptoms of a suspected ADR, and the important role of parents in mediating information about medicines. Children and young people linked symptoms with medicines using temporal association, the absence of a plausible alternative explanations and challenge re-challenge and they described weighing up the risks and benefits of medicines for the future. Children and young people demonstrated a good understanding of the Yellow Card Scheme and wanted to know about, or participate in the reporting of suspected ADRs.

Conclusion Results suggest there is considerable room to enhance communication with children and young people about ADRs. Common ground between how children and young people evaluate ADRs and how parents and clinicians do so suggests a useful starting point. The need for improved communication with parents, who act as mediators, is also indicated. Consideration should be given to how children and young people could be involved in pharmacovigilance for paediatric medicines.

\section{PO-0926 ARE GROUP ASSESSMENTS SUPERIOR TO INDIVIDUAL AVOIDABILITY ASSESSMENTS? A TEST OF THE LIVERPOOL ADVERSE DRUG REACTION AVOIDABILITY ASSESSMENT TOOL}

${ }^{1}$ LE Bracken, ${ }^{1} J C$ Duncan, ${ }^{1} \mathrm{M}$ Peak, ${ }^{2} \mathrm{~J}$ Arnott, ${ }^{3} \mathrm{JJ}$ Kirkham, ${ }^{4} \mathrm{AJ}$ Nunn, ${ }^{5} \mathrm{M}$ Pirmohamed, ${ }^{4}$ MA Turner. 'Research and Development, Alder Hey Children's NHS Foundation Trust, Liverpool, UK; ${ }^{2}$ School of Health, University of Central Lancashire, Preston, UK; ${ }^{3}$ Department of Biostatistics, University of Liverpool, Liverpool, UK; ${ }^{4}$ Department of Women's and Children's Health Institute of Translational Medicine, University of Liverpool, Liverpool, UK; ${ }^{5}$ Department of Molecular and Clinical Pharmacology, University of Liverpool, Liverpool, UK

\subsection{6/archdischild-2014-307384.1547}

Background There is currently no standardised method for determining avoidability of adverse drug reactions (ADRs) and many of the established tools are not suitable for use in paediatrics. We have developed a new tool; the Liverpool ADR avoidability assessment tool (LAAT). Initial testing showed mixed inter-rater reliability.

Aim To test the hypothesis that group assessments are superior to individual avoidability assessments.

Methods Participants were assigned either to a consensus group or to individually assess avoidability for a purposive sample of 20 ADR cases. Individual participants (nurses, pharmacists and doctors) independently assessed the cases. Groups took part in multidisciplinary meetings to assess the cases and reach consensus. The results were compared to the 'gold standard' (the avoidability outcome set by a panel of senior investigators). An 